\title{
Fungal Agents as a Cause of Nasal Polyposis
}

Mohammad Nejadkazem ${ }^{1}$, Alireza Hemati ${ }^{1}$, Saman Vegari ${ }^{1 *}$, Mohammad Adibpour ${ }^{2}$, Leila Davarimajd ${ }^{1}$

1.Department of Otolaryngology, Head \& Neck surgery, Tabriz University of Medical Sciences, Tabriz, Iran

2.Department of mycology, Tabriz University of medical sciences, Tabriz, Iran

\begin{abstract}
Introduction: Sinonasal polyposis is the most common tumor of nasal cavity and sinuses. Its complications are but not limited to sinusitis, breathing difficulties, hyposmia, anosmia and bone erosion.

Methods and materials: A total of 98 patients with sinonasal polyposis were examined for suspicious causative fungal agent.

Results: Direct microscopy and culture confirmed fungal agent in 8 patients (8.1\%) from which 3 cases had Alternaria spp, 1 patient Aspergillus spp, 1 patient Bipolaris spp, and 3 patients yeast. Conclusion: Fungi may be considered as a potential cause of sinonasal polyposis.
\end{abstract}

Keywords: Sinonasal Polyposis, Rhinosinusitis, Fungi

\section{Introduction}

Nasal and sinuses polyposis is the most common tumor of nasal cavity that results from chronic inflammation of nose and sinuses mucosal membrane. Its complications consist of sinusitis, breathing difficulties, hyposmia, anosmia, bone erosion, and sometimes orbital and cranial complications. Sinonasal polyps are common and affect 1-4\% of general population [1]. Although there are some evidences that allergy may be a cause, there are still many questions about the pathogenesis of polyps and their etiology $[2,3]$. It is thought that viral, bacterial and fungal infections and even toxins may be the source of polyps [4-7]. Although recent studies have identified that fungal agents may have a role in polyp formation [8, 9], there are some controversial findings [4]. The most common fungal agents resulting in polyp formation are saprophytic fungal agents such as Alternaria spp, Curvularia, Bipolaris, Crisosporidium, Rhyzomucor and rarely Rhyzosporidium Siberia. Saprophytic fungal agents cause allergic sinusitis and consequent polyposis $[6,10]$. The aim of this study was to

\section{Corresponding author:}

Saman Vegari

Department of Otolaryngology, Head \& Neck surgery, Tabriz University of Medical Sciences, Tabriz, Iran Email: svegari@yahoo.com

Receive date: 2014-02-15| Accept date: 2014-03-18| Publish date: 2014-03-27

DOI: 10.7575/aiac.abcmed.15.03.01.07

\section{Al}


determine the incidence of fungal agents as etiology of sinonasal polyposis.

\section{Materials and methods}

From 2007 to 2012, a total of 98 patients (35 females and 63 males) with the mean age of 34.4 years with sinonasal polyposis were examined in the medical mycology department, Medical university of Tabriz, Iran. All patients underwent surgical polypectomy and all specimens were preserved in sterile conditions. All specimens were examined by direct microscopy after treatment with potassium hydroxide $10 \%$ (KOH , Merk, Germany) and cultured on Sabouraud dextrose agar (Merk , Germany), supplemented with peptone $10 \mathrm{gr}$,
D-Glucose $40 \mathrm{gr}$ and Agar 15 gr. Diagnosis was based on the direct microscopy and then macro and micro morphological features of the colonies. The most common clinical symptoms of the patients were nasal obstruction and rhinorhea.

\section{Results}

Of 98 patients examined, fungus was found in 8 patients ( 3 patients in direct microscopy and culture and in 5 patients only in culture); 6 patients were male and 2 female. Accordingly, in 3 patients Alternaria spp, in 1 patient Aspergillus spp, in 1 patient Bipolaris spp, and in 3 patients yeast were found. The incidence of fungal agents isolated is summarized in Table 1.

\begin{tabular}{llll}
\hline Results & Total (\%) & Male (\%) & Female (\%) \\
\hline Fungus found & $8(8.1)$ & $6(9.5)$ & $2(5.7)$ \\
\hline Fungus not found & $90(91.8)$ & $57(90.4)$ & $33(94.2)$ \\
\hline Total & $98(100)$ & $63(100)$ & $35(100)$ \\
\hline
\end{tabular}

Table 1: The incidence of fungal agents isolated from the patients

mediators and resulting in secondary

\section{Discussion}

Sinonasal polyps, as mentioned above, are the most common tumors of the nasal cavity and sinuses affecting 1-4\% of the populations [1]. Causative agents of sinonasal polyposis, however, remain unknown and even it is not the same in all patients. Allergic fungal rhinosinusitis is a well defined entity of chronic rhinosinusitis and is an IgE-mediated hypersensitivity to fungal agent in nasal cavity and sinuses. Recently, it has been postulated that fungal agents may be the primary cause of chronic rhinosinusitis. According to this concept, inhaled fungi, entrapped in the sinonasal mucosa, cause eosinophilic shift in respiratory mucosa, clustering around and attacking the fungal element, releasing toxic inflammation [8-11]. Fungal infection can cause polyp formation. Therefore, both mechanisms may initiate polyp formation. Management of sinonasal polyps should be based on causative factors; therefore, fungal eradication should be considered if fungal elements are one of the causes of sinonasal polyps and chronic rhinosinusitis $[9,11]$.

In the present study, patients had a history of allergic rhinitis, chronic sinusitis and asthma, so it could be considered that both fungal infection and local allergy to fungal colonization had effect on polyp formation in our patients. In similar studies by standard culture of nasal mucosa, fungal agent had been isolated in 5$95 \%$ in patients with chronic rhiosinusitis, but most of the cultured fungi in these studies were environmental fungi that had possibly 
contaminated the specimen [9]. In our descriptive study, 8 patients had positive culture $(8.1 \%)$ of which in 3 patients Alternaria spp, in 1 patient Aspergillus spp, in 1 patient Bipolaris spp, and in 3 patients yeast were found. Alternaria was seen in direct microscopy and cultured. Aspergilus spp was the most common fungus isolated from nasal polyps in some other studies whereas in some after studies, they were isolated less frequently [12]. Hofman et al. detected Penicillium chrysogeum DNA from $56.5 \%$ of their patients with chronic rhinosinusitis with polyps. $17.4 \%$ of their patients were affected by both Penicillium chrysogeum and Aspergillus fumigates; while, $13 \%$ were negative for DNA tests. This accounted for an $87 \%$ positive result for fungi's DNA in patients with sinus polyps [13]. The latter ratio was reported $78.2 \%$ by Arauio et al. [4].

Our findings could be related to environmental factors and geographic differences in detection of fungal species from nasal polyps. However, future studies are advocated to determine the precise role of fungal agents in development of sinonasal polyps.

\section{Acknowledgments}

This research was supported by Tabriz University of Medical Sciences, Tabriz, Iran. We would like to thank mycology research and otolaryngology and head and neck surgery departments' staff and physicians.

\section{Conflicts of interest}

The authors declare that they have no conflict of interest.

\section{References}

1. Batman ND, Fahy C, Wollford TJ (2003). Nasal polyps: still more questions than answers. J laryngology Otol, 117(1): $1-9$.

2. Bernstein JM, Kansal R (2005). Superantigen hypothesis for the early development of chronic hyperplastic sinusitis with massive nasal polyposis. Curr Opin Otolaryngol Head Neck Surg, 13(1): 39-44.

3. Blitzer A, Lawson W(1993). Fungal infections of the nose and paranasal sinuses, Part I. Otolaryngol Clin Norh Am, 26(6): 1007-35.

4. Elizabeth Araujo, Celso Dall Igna, Fabio Anselmi, Bruno Carlos Palombini(2003). Prevalance of patients with fungi in chronic rhinosinositis. Otolaryngology- Head and neck surgery. 131(2): 267.

5. Kupferberg SB, Bent JP, Kuhn FA(1997). Prognosis for allergic fungal sinusitis. Otolaryngology Head and Neck Surg, 117: $35-41$.

6. Pitzurra L, Bellocchio S, Nocentini A, Bonifazi P, Scardazza R, Gallucci L, et al (2004). Antifungal immune reactivity in nasal polyposis. Infection and Immunity,72(12): 7275-81.

7. Muzaffar M, Luqman M, Nabi Gh, Malik IA, Ullah Kh (1990). Aspergillus granuloma presenting as recurrent nasal polyp. Tropical Doctor, 20: 95-96.

8. Weschta M, Rimek D, Formanek M, PolzehI D, Riechelmann H (2003). Local production of Aspergillus fumigatus. specific immunoglobulin E in nasal polyps.The American Laryngological, Rhinological \& Otological Society, Inc 113(10): 1798-02.

9. Norlander T, Bronnegard M, Stierna P(1999). The relationship of nasal polyps, infection and inflammation. Am J Rhinol, 13(5): 349-55.

10. Ricchetti A, Landis BN, Maffioli A, Giger R, zeng, Lacroix JS (2002). Effect of anti-fungal nasal lavage with amphotericin B on nasal polyposis. J Laryngol Otoll, 116 (4): 261-3. 
11. Richardson MD, Warnock DW (2003). Fungal infection: Diagnosis and management. 3rd ed. Blackwell. Massachusetts. pp.169, 180-1, 340.

12. Rupa V, Jacob M, Mathews MS (2001). Atopy, proptosis, and nasal polyposis. Postgrad Med J, 77:343-4.

13. Hofman A, Hofman T, Skrobisz W(2007). The Role Of Fungi In Sinus Polyposis . Journal of Allergy and Clinical Immunology, Volume 119, Issue 1, Pages S141-S141. 